Historic, Archive Document

Do not assume content reflects current scientific knowledge, policies, or practices. 


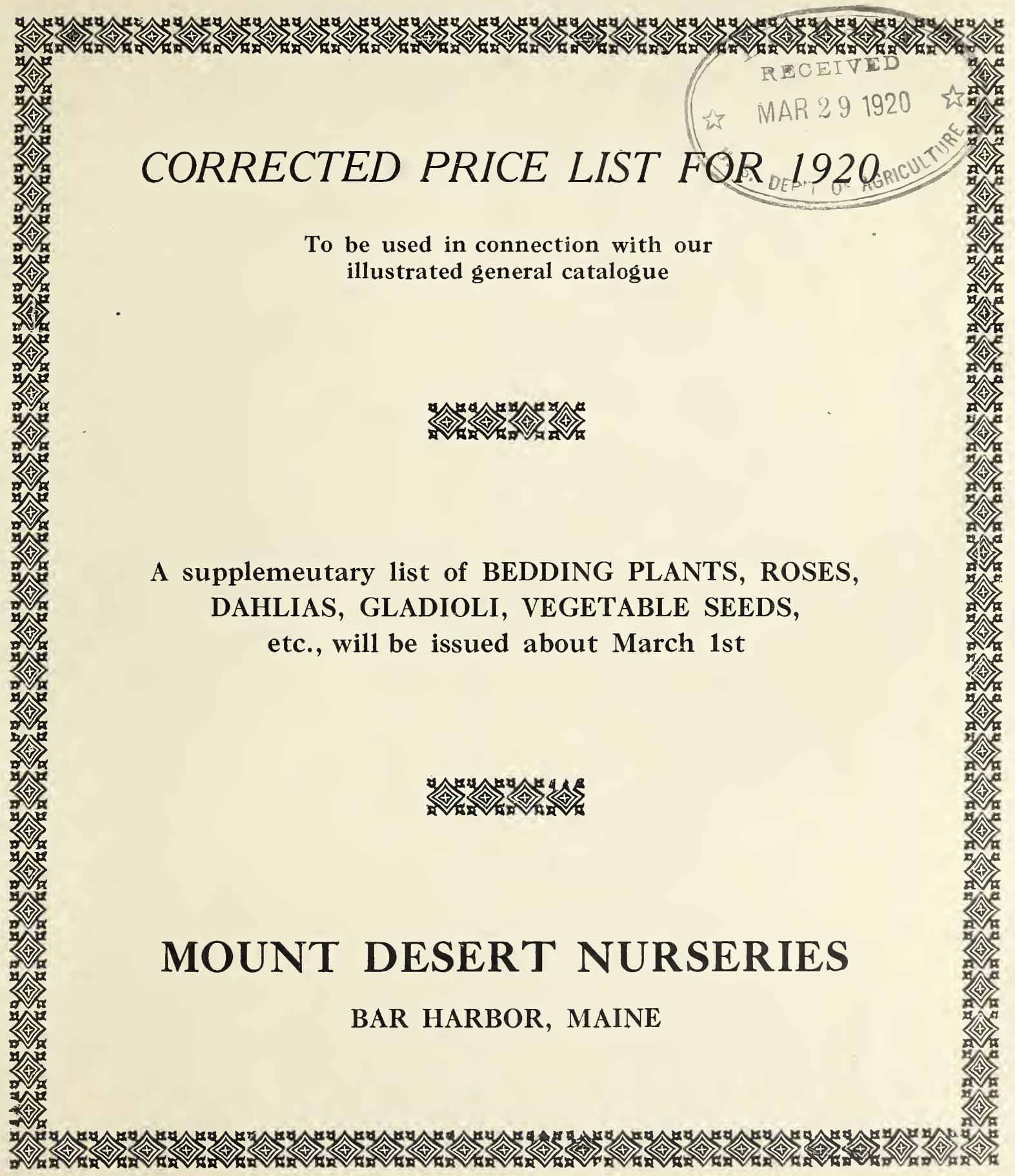





\section{PLANTS OF SPECIAL MERIT}

The corrected prices of these plants will be found in their regular order in the following corrected general price list.

\section{ALPINE PLANTS}

All plants in this list will be furnished at the uniform rate of 25 cents each; $\$ 2.00$ per 10 .

Other Alpine plants not named in general list: Acaena argen tae

Alyssum saxatile compactum

Armeria laucheana alba

Armeria laucheana rosea

Campanula glomerata acaulis

Dianthus caesius

Erysimum rupestre

Gentiana Przewalski

Gypsophila repens

Linum perenne

Tunıca saxifraga

Veronica longifolia

\section{HARDY HERBACEOUS PLAN'TS}

ACHILIE each Cerise Queen........

ACONITUM acutum......... .35

autumnale....... .35

barbatum ........ $\quad .35$

exaltatum........ $\quad .35$

cammarum....... $\quad .35$

cammarum Stoerkianum .35

Fischeri......... . 35

napellus......... .35

napellus album..... $\quad .35$

napellus bicolor ... . $\quad .35$

napellus Meilichoferi.

volubile tenuisectum

Wilsoni............

AGROSTEMMA coronaria .......

ANGHUSA italica Dropmore . . . . .

italica Opal . . . . . . . . .

A QUILEGIA, Long Spurred Hybrids

ARTEMISIA pontica ..........

frigida.

gnaphaloides.......

stellariana.........

lactiflora per 10

2.00

3.00

3.00

3.00

3.00

3.00

3.00

3. 00

3. 00

3.00

3.00

3.00

4.00

4.00

2.00

3.00

3.00

2.00

2.50

2.50

2.50

2.50

3.00
3.00
ASTER, Avalanche

Climax

Glory of Colwell

Novae Angliae, Lil Fardel. .

Novae Angliae, William

Bowman............

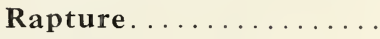

St. Egwin.

subcoerulea

ASTILBE Arendsi

Ceres.

Gloria.

Gruno

Juno.

Moerheimi

Rose Perle.

Salland

Salmon Queen

Silver White...

Venus........

Vesta.........

White Pearl....

chinense ...........

Davidi

hybrida Avalanche.

Marguerite.....

Meta Immink.

Peach Blossom.

Queen Alexandra

Thunbergi delicata . . . .

BOLTONIA asteroides..........

latisquama...

BUDDLEIA magnifica

superba

(Large plants 1.00 to 1.50 each)

CAMPANULA latifolia alba......

latifolia macrantha.

medium (Canterbury

Bells)

persicifolia grandi-

flora.

persicifolia grandi-

flora alba ..........

persicifoli..........

rapunculoides

GENTAUREA montana . 
CHELONE Lyoni .

CHR YSANTHEM UM King Edward uliginosum.

CIMICIFUGA cordifolia

japonica

.

simplex

CLEMATIS integrifolia coerulea

CONVALLARIA, Berlin Crowns,

$$
\text { (pips) per } 100 \$ 5.00
$$

Fortin's Giant(pips)

DELPHINIUM Belladonna........

Clivedon Beauty...

formosum.

formosum coelestinum

hybrids mixed....

hybrids Moerheimi.

DICENTRA sepctabilis .

DICTAMNUS caucasicus

$$
\text { fraxinella.......... }
$$

DIGITALIS gloxinaeflora (Foxgloves) . . . . . . . . . . . .

lutea.................

DORONICUM plantagineum excelsum

ECHINOPS ritro

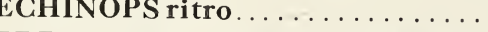

EREMURUS. Prices and sizes on application.

ERR YNGIUM planum ........ . . 35

FUNKIA, in variety. . . . . . . . . .35

GILLENIA trifolia ta . . . . . . . . . . .35

GYPSOPHILA paniculata ...... .35

HELENIUM autumnale pumilum

$$
\begin{gathered}
\text { compact } \\
\text { pumilum }
\end{gathered}
$$

magnificum

HELENIUM autumnale superbum

HELENIUM Hoopesi............

HELIANTHUS, Miss Mellish . . . . . .

rigidus semiplenus
strumosus.........

HELIOPSIS laevis Pitcheriana .....

HEMEROCALLIS aurantiaca .... .

Dumortieri.....

fulva.

hybrida Gold Dust

hybrida lu teola...

hybrida luteola

pallens.

hybrida Orange Man
35

.35

.35

.35

.35

.75

.35

2.50

2.50

2.50

2.50

2.50

2.50

2.50

2.50

.75

.75

2.50

2.50

2.50

2.50

2.50

3.00

3.00

3.00

3.00

3.00

2.50

2.50

2.50

2.50

2.50

2.50

2.50

2.50

.25

.25

.25

.25

.25

.25

.25

.35

.35

.35

.35

.35

.35

.35
2.00

2.00

2.00

2.00

2.00

2.00

2.00

2.50

2.50

2.50

2.50

2.50

3.00

2.50 hybrida Sovereign

Middenorfi......

minor .........

Thunbergi.....

HESPERIS matronalis..........

HOLLYHOCKS, Double. In separate

varieties.........................

INCARVILLEA Delavayi . . . . . . . $\quad .35$

IRIS germanica

New varieties, as per catalogue... $\quad .35$

Older varieties, as per catalogue . $\quad .25$

IRIS kaem pf eri

Varieties, as per catalogue......

LATH YRUS lati folius, white pearl.

LIATRIS callilepis

pycnostachya..........

LUPINUS polyphyllus .

polyphyllus albus.

polyphyllus Moerheimi.

LYSIMACHIA clethroides ........

MONARDA calmonea (New) ......

didyma, Cambridge

Scarlet.

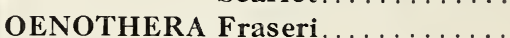

fruticosa major....

Youngi .

PACHYSANDRA terminalis $\ldots .$.

PAPAVER nudicaule .....................

orientale . . . . . . .

PEONIES, Single:

Hyperbolus

Lady Wimbourne

Oniverie

Stanley

Semi-Double:

Bridesmaid

Jessieu

Mt. Kebo

Pemetic

Double:

Alba Sulphurea

Armandine Mechin

Charles Verdier

Claire du Bois

Couronne d'Or

Delachi

Duchesse de Nemours

Duchesse d'Orleans

Duchess of Teck
2.50

2.50

2.50

2.50

2.00

2.50

2.50

3.00

2.00

2.50

2.50

2.50

2.50

2.50

2.50

2. 50

2.50

3.00

2. 00

2.50

2.00

2.00

2.00

1.50

.20

.35

2.50 


\section{Duke of Wellington \\ Eden \\ Felixe Crousse \\ Festiva Maxima \\ Fushimi \\ Humei \\ Jeromus \\ Jules Calot \\ Kelway's Queen \\ Lady Beresford \\ Lady Carrington \\ Lord Rosebery \\ Mme. Chaumy \\ Mme. Lebon \\ Marie Lemoine \\ President Roosevelt}

Any of the above............ $\quad .75 \quad 6.00$

Four-year-old clumps.......... $2.50 \quad 20.00$

PHLOX, Beacon

Caran d'Ache

Cigale

Esperance

Elizabeth Campbell

Eugene Danzanvilliers

Europa

Faust

Frau Ant. Buchner

Fraulein Von Lassberg

Gen. Chanzy

Gen. Van Heutz

Goliath

Gruppenkonigin

Iris

Josephine Gerbeaux

Le Mahdi

Louise Abbema

Miss Lingard

Mrs. Charles H. Dorr

George B. Dorr

Pantheon

Rijnstreom

Selma

R. P. Struthers

Sylphide

Von Hochberg

Any of the above
PLATYGODON grandiflorum...... . .25 2.00

$\begin{array}{lll}\text { grandiflorum album . . } & .25 & 2.00\end{array}$

POLYGONATUM japonicum....... . .25 2.00

multiflorum..... $.25 \quad 2.00$

POTENTILLA, in variety . . . . . . . . . . $\quad .25 \quad 2.00$

PYRETHRUM, Aphrodite......... . .35 3.00

Figaro........... . $35 \quad 3.00$

Lord Rosebery ....... . .35 3.00

Mme. Munier........ $\quad .35 \quad 3.00$

Queen Mary......... $\quad .35 \quad 3.00$

Yvonne Cayeux...... $\quad .35 \quad 3.00$

Rudbeckia lacinia ta plena ......... . . $25 \quad 2.00$

Newmani............ .25 2.00

nitida, Autumn Glory. . . . . $\quad .25 \quad 2.00$

speciosa.............. .25 2.00

subtomentosa ......... .25 2.00

SENEGIO Glivorum.............. .25 2.00

Veitchianus........... . $35 \quad 3.00$

Wilsonianus ........... $.35 \quad 3.00$

SIDALGEA oregana............. . .35 2.50

SPIRAEA filipendula ............ . $35 \quad 2.50$

filipendula plena........ . $35 \quad 2.50$

gigantea rosea.......... . $35 \quad 2.50$

kamtschatica.......... . $35 \quad 2.50$

purpurea..................... $35 \quad 2.50$

purpurea alba......... . .35 2.50

purpurea elegans........ $\quad .35 \quad 2.50$

rubra.................... $35 \quad 2.50$

rubra var. venusta....... . $35 \quad 2.50$

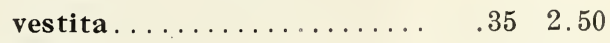

STATICE altaica.............. .35 2.50

latifolia........................ $35 \quad 2.50$

tartarica........... .35 2.50

SWEET WILLI AM, in variety . . . . . . . .25 2.00

THALICTR UM aquilegifolium...... . .25 2.00

\dipterocarpum..... .50 4.00

glaucum.......... . $35 \quad 2.50$

I

$.35 \quad 2.50$

$\begin{array}{llrr}\text { THERMOPSIS caroliniana .......... } & .35 & 2.50 \\ \text { TRADESGANTIA virginiana . . . . . . . } & .25 & 2.00\end{array}$

TROLLIUS asiaticus . . . . . . . . . . .35 2.50

asiaticus flore croceo..... $\quad .35 \quad 2.50$

caucasicus, Orange Globe.. $\quad .35 \quad 2.50$

Gibsoni................... $\quad .35 \quad 2.50$

hybrids, named varieties.... . .35 2.50

japonicus Excelsior...... . . $35 \quad 2.50$

japonicus plenus....... . $35 \quad 2.50$

napellifolius ........... . $35 \quad 2.50$ 
VERONICA bachofensis longifolia subsessilis spicata................

YUCGA filimentosa

\section{BULB LIST}

GALTONIA candicans. Price on application.

IRIS xiphioides (English) (Out of stock for 1920) xiphium (Spanish) Out of stock for 1920

\begin{tabular}{|c|c|c|}
\hline & & \\
\hline LILIUM auratui & .60 & 5.0040 .00 \\
\hline candidum ....... & .25 & 3.0025 .00 \\
\hline elegans atrosanguineum & .30 & 2.5020 .00 \\
\hline Hansoni............ & .40 & 3.0025 .00 \\
\hline$\ldots \ldots \ldots \ldots \ldots$ & .75 & 6.00 \\
\hline speciosum album ..... & .50 & 4.00 \\
\hline speciosum roseum.... & .50 & 4.00 \\
\hline speciosum rubrum.... & .50 & 4.00 \\
\hline speciosum magnificum. & .50 & 4.00 \\
\hline superbum......... & .35 & 3.00 \\
\hline tigrinum splendens.... & .25 & 2.2520 .00 \\
\hline NTBRETIA, Ernest Davison. & & 1.2510 .00 \\
\hline George Davison. & & 1.2510 .00 \\
\hline Germania..... & & 1.2510 .00 \\
\hline King Edmund. . & & 1.2510 .00 \\
\hline Vesuve........ & & 1.2510 .00 \\
\hline Westwick...... & & 1.2510 .00 \\
\hline
\end{tabular}

NARCISSUS. Prices quoted in August for fall delivery. SNOWDROPS. Prices quoted in August for fall delivery TULIPS. Prices quoted in August for fall delivery.

\section{AQUATICS AND BOG PLANTS}

ACORUS. Out of stock for 1920.

APONOGETON. Out of stock for 1920.

ASTILBE. See general list.

CALTHA. Out of stock for 1920.

IRIS, Kaempferi

Monnieri

pseudacorus

Sibirica

Sibirica alba. .

maxima

orientalis

orientalis Snow Queen

For any except the Kaempferi........ . .25 2.00

LOBELIA cardinalis ............. .25 2.00
LYTHRUM roseum............ .25 2.00

NYMPHAEA. .Prices and varieties on application.

SAGITTARIA japonica flore pleno.... . .50 4.00

SAGITTARIA sagit taefolia ......... . $35 \quad 3.00$

SENEGIO Glivorum ............. .25 2.00

Veitchianus........... .35. 3.00

Wilsonianus ........... $\quad .35 \quad 3.00$

\section{ROSES}

HYBRID PERPETUALS:

Baroness Rothschild ........... .90 $\quad 7.50$

Clio (Out of stock for 1920) .........

Duke of Edinburgh (Out of stock for 1920)

Frau Karl Druschki (See Hybrid Teas). $\quad .90 \quad 7.50$

General Jacqueminot (Out of stock for 1920)

Hugh Dickinson. . . . . . . . . . . .90 7.50

Madam Gabriel Luizet (Out of stock for 1920)

Margaret Dickson (Out of stock for 1920)

Mrs. John Laing. . . . . . . . . . . . . . . .90 $\quad .50$

Paul Neyron............... .9. $90 \quad 7.50$

Ulrich Brunner. . . . . . . . . . . .90 7.50

HYBRID TEAS :

Bessie Brown (Out of stock for 1920)

Caroline Testout ............. .9 $\quad .90 \quad 7.50$

Chrissie MacKeller. . . . . . . . . . .90 $\quad 7.50$

Dorothy Page Roberts (Out of stock for 1920)

Etoile de France . . . . . . . . . . . . . .90 $\quad 7.50$

Frau Karl Druschki............. . .90 $\quad 7.50$

George Arends............... .90 $\quad .50$

Gruss an Teplitz (Out of stock for 1920)

Kaiserin Augusta Victoria.......... $\quad .90 \quad 7.50$

Killarney................ .90 7.50

Madam Abel Chatenay (Out of stock for 1920)

Maman Cochet (Out of stock for 1920)

Maman Cochet White (Out of stock for 1920)

Miss Kate Moulton . . . . . . . . . . . . .90
American Pillar............... 1.00

Dorothy Perkins............... 1.00

Dorothy Perkins White.......... 1.00

Hiawatha.................. 1.00

Lady Gay . . . . . . . . . . . . . . . 1.00

La Fiamma ................. 1.00

Lucille ....................... 1.00

Tausendschon................. 1.00

Trier........................ 1.00

Paradise...................... 1.00 


\section{MOUNT DESERT NURSERIES}

Wichuraiana

1.00

Out of Stock for 1920 :

Crimson Rambler

Setigera

Dawson

Fairy Cup

Farquhar

Prairie Queen

NOTE: The above Climbing Roses are two-year-old, grown one year in the open ground and one year in pots. They will be sold from five and six inch pots.

NOTE: Our Deciduous Trees, Evergreen Trees and Shrubs, Deciduous Shrubs, Vines and Climbers, and FRUITs will be sold according to size and quality of stock selected. We will be very glad to quote on any list of requirements.

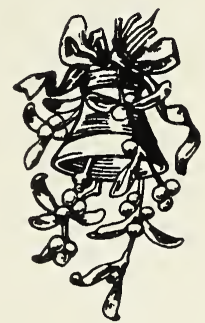


